OPEN ACCESS

Edited by:

Peggy D. McCardle, Peggy McCardle Consulting, LLC and Haskins Laboratories, United States

Reviewed by:

Sabrina E. B. Schuck, University of California, Irvine, United States Kristine Ann Kovack-Lesh Ripon College, United States

*Correspondence: Darlene A. Kertes dkertes@ufl.edu

Specialty section: This article was submitted to Developmental Psychology, a section of the journal

Frontiers in Psychology

Received: 24 May 2018

Accepted: 26 July 2018

Published: 05 September 2018

Citation:

Kertes DA, Hall N and Bhatt SS (2018) Children's Relationship With Their Pet Dogs and OXTR Genotype Predict Child-Pet Interaction in an

Experimental Setting

Front. Psychol. 9:1472. doi: 10.3389/fpsyg.2018.01472

\section{Children's Relationship With Their Pet Dogs and OXTR Genotype Predict Child-Pet Interaction in an Experimental Setting}

\author{
Darlene A. Kertes ${ }^{1,2 *}$, Nathan Hall ${ }^{3}$ and Samarth S. Bhatt ${ }^{1}$ \\ ${ }^{1}$ Department of Psychology, University of Florida, Gainesville, FL, United States, ${ }^{2}$ Genetics Institute, University of Florida, \\ Gainesville, FL, United States, ${ }^{3}$ Department of Animal and Food Sciences, Texas Tech University, Lubbock, TX, United States
}

Human-animal interaction (HAl) research has increasingly documented the important role of pet dogs in children's lives. The quality of interaction between children and their pet dogs, however, is likely influenced by individual differences among children as well as their perceived relationship with their pet dog. Ninety-seven children aged 7-12 years and their pet dogs participated in a laboratory protocol during which the child solicited interaction with their dog, from which time petting and gazing were recorded. Children reported on their perceived relationship with the pet dog via interview. Children provided saliva samples, from which a polymorphism in the oxytocin receptor, OXTR rs53576, which has long been implicated in social behavior, was genotyped. The results showed that OXTR genotype and children's perceived antagonism with the pet dog predicted the amount of petting, but not gazing, between children and their pet dogs. This research adds to the growing body of HAl research by documenting individual differences that may influence children's interactions with animals, which is key to research related to pet ownership and understanding factors that may impact therapeutic interventions involving HAl.

Keywords: human-animal interaction, relationships, child, petting, dogs, OXTR, oxytocin receptor gene, oxytocin

\section{INTRODUCTION}

Human-animal interaction (HAI) research has increasingly documented the important role of pet dogs in providing social support to children (e.g., Friedmann et al., 1983; Kotrschal and Ortbauer, 2003; Anderson and Olson, 2006). However, the bulk of this research has been descriptive or correlational in nature, with long-standing concerns about methodological rigor (Griffin et al., 2011) and few well-controlled laboratory experiments that afford greater confidence to the validity of results (Wilson and Barker, 2003). Our group has previously reported that children randomly assigned to experience a standard laboratory stressor accompanied by their pet dog for social support reported feeling less stressed compared to children who completed the stressor with their parent present or with no social support (Kertes et al., 2017). Among the children who underwent the stressful experience with their pet dog, those who naturally solicited their dog to be stroked or petted had lower levels of the stress-sensitive hormone cortisol compared to children who engaged their dog less. 
Indeed, petting, and to a lesser extent gazing, have been suggested as potential mechanisms by which HAI impact humans by altering their emotional and physiological state (e.g., Odendaal and Meintjes, 2003; Shiloh et al., 2003). Among adults, petting is associated with reduced perceived stress or anxiety (Shiloh et al., 2003; Barker et al., 2005), increased immunoglobulin A (Charnetski et al., 2004), lower heart rate or blood pressure (Jenkins, 1986; Vormbrock and Grossberg, 1988; Demello, 1999; Handlin et al., 2011), and changes in $\beta$-endorphins, prolactin, $\beta$-phenylethylamine, oxytocin, cortisol, and dopamine (Odendaal, 2000; Barker et al., 2005). Adult owner-pet gazing has been linked with increased oxytocin levels (Nagasawa et al., 2009, 2015). Among children, petting during child-dog interaction has been associated with lowered cortisol stress response (Kertes et al., 2017) and positive affect (Kerns et al., 2018). The vast majority of research on HAI with children has focused on dog presence, which has been linked with reduced blood pressure (Friedmann et al., 1983) perceived stress (Kertes et al., 2017), enhanced emotional stability in the classroom (Anderson and Olson, 2006), increase social interaction, and decrease aggression and hyperactivity (Kotrschal and Ortbauer, 2003), and reduced distress to a routine medical procedure (Vagnoli et al., 2015).

Its potential benefits notwithstanding, the degree to which children's interactions with their pet dogs spontaneously include petting and gazing may be influenced by individual differences in children's perceived relationship with their pet dog. To date, the majority of research on pet owners' feelings toward their pets have centered on positive emotions (Johnson et al., 1992; Cromer and Barlow, 2013). This area of research has shown that children and adults alike report strong positive feelings toward their pet dogs (Serpell, 1996; Daly and Morton, 2006; Kurdek, 2008). Noticeably absent from most HAI studies is the role of perceived negative aspects of the child-pet relationship, such as feeling annoyed with or hassled by interactions with the pet. A more complete evaluation of effects of children's feelings toward their pet necessarily involves inclusion of both positive and negative components to children's perceived relationships (e.g., DeRosier and Kupersmidt, 1991; Furman and Buhrmester, 1992; Shantz and Hartup, 1992; Van Horn and Cunegatto, 2000; Moilanen and Raffaelli, 2010).

Another factor that may contribute to individual differences in children's interactions with their pet is variability within the oxytocinergic system. Oxytocin is a hormone and neuromodulator shown to be involved in a variety of social behaviors (Carter, 2014). Oxytocin is linked with affiliative behavior (Insel, 1992), formation of social bonds (Lim and Young, 2006), and responses to stressful social situations (Neumann et al., 2000; Kirsch et al., 2005).

Endogenous circulating oxytocin effects are influenced by actions at the oxytocin receptor. This receptor is encoded by the gene OXTR, which is variably expressed across individuals. Among the most commonly studied genetic polymorphisms at OXTR is rs53576, involving a guanine $(\mathrm{G})$ to adenine (A) substitution. Genetic variation at this locus has been associated with both prosocial and negative behaviors. For example, A carriers, (i.e., individuals with the AA or AG genotype), compared to those with the GG genotype, have demonstrated lower levels of interpersonal empathy (Rodrigues et al., 2009; Smith et al., 2014), trust (Krueger et al., 2012), as well as lower self-esteem (Saphire-Bernstein et al., 2011), and higher negative affect and loneliness (Lucht et al., 2009). Among adolescents, A-carriers are reported to be less responsive to parental support (Smearman et al., 2016). Consistent with the differential susceptibility hypothesis (Belsky et al., 2009), it has been suggested that OXTR rs53576 may be one of a set of susceptibility loci in the genome, whereby genetic variation influences an individual's sensitivity to the social environment (Kim et al., 2010).

Notably, the extensive literature examining OXTR rs53576 in relation to social behavior has focused exclusively on human social interaction. To date, there are no published studies examining OXTR genotype with respect to children's interaction with animals. This is notable because interaction with and ownership of lay or trained therapy dogs is increasingly becoming a mainstay of clinical interventions for children with anxiety disorders, autism spectrum diagnoses, or a history of maltreatment, on the assumption that interacting with animals is particularly beneficial for individuals for whom social interactions are challenging (Nimer and Lundahl, 2007). The present report is the first to assess whether the OXTR genotype among children is related to HAI.

This study focused on typically developing children in middle childhood (aged 7-12 years). During this developmental period, the amount of time children spend with parents declines dramatically compared to earlier ages (Lam et al., 2012). Although parents continue to be important social partners, in middle childhood, children begin to rely on a broader network of social support figures compared to earlier ages, including pets (Bryant, 1985).

The purpose of the present study was to test whether children's perceived relationships (including both positive emotional support and negative interactions) along with child genotype at the OXTR rs53576 polymorphism predict directly observable child-pet interaction. To achieve this aim, we assessed two essential elements of child-pet interaction, petting, and gazing, via direct behavioral observation in the context of a controlled laboratory environment with minimal distractions. Based on the extant literature linking OXTR rs53576 genotype to social behavior, we expected that children who are A-carriers would differ from those with the GG genotype with respect to the amount of time spent petting and gazing with their pet dogs. Because of the complex associations of the OXTR genotype with respect to social behavior, and the fact that this is the first study to examine the rs53576 polymorphism with respect to HAI rather than human social interactions, we did not specify a priori directional predictions for the OXTR genotype. With respect to children's relationships, we anticipated that children's perceived relationship with their pet dogs, as reflected in higher levels of perceived support and lower levels of perceived negative interactions, would be associated with higher levels of petting and gazing with their pet dogs. Children were also asked about their perceived relationship with the mother, which was included on conceptual grounds for their key role in social emotional 
development, and to evaluate whether child-parent relationships were related to child-pet interactions.

\section{MATERIALS AND METHODS}

\section{Participants}

Participants were 97 children (49 boys; 48 girls) accompanied by a parent $(81 \%$ mother) and pet dog. Participating families were recruited through locally distributed mailings, flyers, and radio and TV advertisements. Interested families contacted the research lab and were screened for eligibility. To be eligible for the study, children could not have any diagnosed physical or behavioral health conditions, and the pet dog must have lived with the family for at least 6 months and have no history of aggression. If multiple dogs resided in the home that met inclusion criteria, the family selected one dog based on the child-pet relationship to accompany the child to the research lab.

Child age ranged from 7 to 12 years $(M=10.3$ years, $S D=1.32)$ Child ethnicity was reported by parents as follows: $11 \%$ Hispanic; $89 \%$ non-Hispanic. The majority of the sample was White (84\%), with the remainder reporting their race as follows: $7 \%$ two or more races; $3 \%$ Latino; $2 \%$ Native American; 2\% African American, and 2\% Asian.

\section{Procedure}

Procedures were approved by the University of Florida Institutional Review Board and the Institutional Animal Care and Use Committee. All procedures took place in three adjacent rooms - a waiting room, interview room, and experimental testing room - at the research laboratory at the University of Florida. Children were aware of their parent's and dog's location at all times. All rooms were temperature controlled and water was available for the dog.

At the start of the study visit, parents provided written informed consent in the waiting room. The study was also explained to children verbally for purposes of oral assent. A trained dog handler brought the dog to the experimental testing room to familiarize the dog to the room and study personnel. Then, the child accompanied an experimenter to the interview room, decorated in child-friendly décor while the dog remained with the parent in the waiting room.

In the waiting room, parents completed questionnaires providing basic demographic information on their child, family, and pet dog. Parents also provided information about the breed of the dog, which was subsequently classified into breed groups. Children's pets included lap/toy dogs $(n=31)$, sporting breeds ( $n=20)$, herders $(n=18)$, terriers/ratters $(n=12)$, bully/fighting breeds $(n=11)$, and unknown mixes $(n=5)$. A research assistant was present throughout to answer any parent questions.

\section{Children's Perceived Relationships}

In the interview room, children completed an experimenterassisted questionnaire about their relationships with their mother and their pet dog using the Network of Relationships Inventory (NRI) (Furman and Burmheister, 1985). The original NRI, comprised of 21 items, was designed and has been validated for assessing relationship qualities across a broad variety of social relationships, including but not limited to parents, teachers, and peers. An example item from this measure is, "How often do you tell this person everything that you are going through?" The measure contains 10 subscales typically collapsed into two broader scales, termed Support and Negative Interactions. We have previously evaluated the NRI among children owning pet dogs to determine the relevance of items for assessing child-pet relationships. With the exception of two subscales, Instrumental Aid and Conflict, the remaining subscales were retained as applicable to child-pet relationships (Hall et al., 2016).

The NRI items tapping relationship with the mother was scored as recommended by the scale developers (Furman and Burmheister, 1985). Then, the overarching dimension of Support was created by computing the mean of the items on the subscales Companionship, Intimate Disclosure, Nurturance, Affection, Admiration, Reliable Alliance, and Instrumental Aid. The dimension of Negative Interactions was computed as the mean of the scores on Conflict and Antagonism.

The NRI pet items were subjected to a principal component analysis (PCA) to determine whether a two-dimension solution was appropriate with the more limited set of subscales assessed for child-pet relationships. As described in the Section "Results" a two-dimension solution was deemed appropriate for the data, and therefore the subscale means were computed and averaged into the two broad dimensions as follows: Support (Companionship, Intimate Disclosure, Nurturance, Affection, Admiration, Reliable Alliance) and Negative Interactions (Antagonism).

\section{Behavioral Assessment of Child-Pet Interaction}

The child and pet dog were brought to the experimental testing room for behavioral assessment of interaction between the child and pet. Specifically, this assessment measured the proportion of time the child and dog spent interacting while the child was sitting quietly in a room $(4.5 \mathrm{~m}$ by $3 \mathrm{~m})$ that contained a chair, desk, and lamp for $10 \mathrm{~min}$. This task was based on components of past sociability tests (e.g., Barrera et al., 2010; Jakovcevic et al., 2012), but were simplified such that the child could implement the protocol with brief instruction. The child sat in a chair in the center of a $1 \mathrm{~m}$ radius semi-circle marked with tape on the floor. The dog handler brought the dog to the opposite end of the room, where the dog was able to greet a second observer for approximately $1 \mathrm{~min}$ before beginning the task. The child was asked to direct attention to the dog, and call the dog over twice while remaining seated, once at the beginning of the $10 \mathrm{~min}$ session and again after the first $5 \mathrm{~min}$. The child was asked to otherwise remain neutral unless the dog entered the semi-circle. If the dog entered the circle the child was told to interact with it as if they were at home, to capture natural variability in childpet interaction. During the assessment, the handler and observer stood along the wall opposite of where the child was seated for an unobstructed view of the child-pet interaction.

The handler and the observer, both trained in coding dog behaviors, scored each session live on two dimensions: gazing and petting. Each behavior was scored using partial-interval 
recording by breaking the $10 \mathrm{~min}$ session into $1205 \mathrm{~s}$. epochs. If a target behavior occurred during that epoch, the interval was scored. The percentage of epochs during which a target behavior occurred was averaged across the scorers. Gazing was defined as the percentage of intervals in which the dog and child were facing each other. Petting was defined as the percentage of intervals in which the child made contact with the dog with their hand. Interclass correlations among the two coders was 0.85 for gazing and 0.99 for petting.

\section{Genotype Assessment}

Children were asked to provide a $4 \mathrm{~mL}$ saliva sample by passive drool into Oragene-DNA (OGR-500) saliva collection tube (DNA Genotek, Kanata, ON, Canada), which was stored at room temperature until the DNA extraction step. DNA extraction was performed using our lab's standardized protocol. Briefly, $750 \mu \mathrm{l}$ of the content from OGR-500 tube was incubated at $50^{\circ} \mathrm{C}$ in a GeneMate dry bath (Bioexpress, Kaysville, UT, United States) for $2 \mathrm{~h}$, followed by incubation on ice for $10 \mathrm{~min}$ and centrifugation at $21,100 \times g$ for $10 \mathrm{~min}$. The DNA was precipitated by transferring the supernatant to a tube containing $750 \mu \mathrm{l}$ of ethanol, mixing the content gently, incubating at room temperature for $10 \mathrm{~min}$, and centrifuging at $16,000 \times g$ for 3 min. The DNA pellet was washed using $70 \%$ ethanol, dried at room temperature, dissolved in $100 \mu \mathrm{l}$ TE buffer and stored at $-80^{\circ} \mathrm{C}$. The DNA quality and quantity was measured using NanoDrop 2000 spectrophotometer (Thermo Fisher Scientific, Wilmington, DE, United States). Genotyping was performed using TaqMan Genotyping Master Mix (Applied Biosystems, Foster City, CA, United States), TaqMan SNP Genotyping assay (Applied Biosystems, Foster City, CA, United States) for OXTR (rs53576), with the StepOnePlus real time PCR system (Applied Biosystems, Frederick, MD, United States), according to the manufacturer's instructions. PCR was performed using 10:1 reaction mix with $1.5 \mathrm{ng}$ DNA and the following cycling conditions: $60^{\circ} \mathrm{C}$ for $30 \mathrm{~s}, 95^{\circ} \mathrm{C}$ for $10 \mathrm{~min}, 40$ cycles of $95^{\circ} \mathrm{C}$ for $15 \mathrm{~s}$ and $60^{\circ} \mathrm{C}$ for $1 \mathrm{~min}, 60^{\circ} \mathrm{C}$ for $30 \mathrm{~s}$. Allelic discrimination was performed using StepOne v.2.1 (Applied Biosystems, Frederick, MD, United States).

\section{Statistical Analysis}

Statistical analyses were conducted in $\mathrm{R}$ version 3.3.2 (R Core Team, 2016). A hierarchical regression framework with backward selection was utilized to test for significant predictors of two dimensions of child-pet interaction, with predictors of petting and gazing tested in separate models. Tested predictors included demographics, relationship qualities, and child genotype.

\section{RESULTS}

\section{Evaluating the NRI to Assess the Child-Dog Relationship}

We first calculated the mean scores for each subscale for the child-dog relationship. We then conducted Principal Component Analysis on the scaled scores of each subscale. Two components explained $71 \%$ of the variance (55 and $16 \%$ respectively). The loadings of each component are shown in Table 1. Inspection of the loadings suggests a two-factor solution identical to Support and Negative Interactions used for computing summary scores for the child-mother relationship. This suggested that these two summary scores can be computed similarly for the child-dog relationship.

\section{Descriptives}

Descriptive statistics of child-reported relationship qualities and behavioral observation of child-pet interaction are shown in Table 2. Genotype assessment of rs53576 yielded the following genotypes: $41 \% \mathrm{GG}, 53 \% \mathrm{AG}$, and $6 \% \mathrm{AA}$. These proportions are comparable to other U.S.-based studies (see for review Luo and Han, 2014). As is common in analysis of rs53576, the AG, and AA genotypes were combined into one genotype group, termed A-carriers, yielding two genotype groups for analysis, GG homozygotes, (41\%) and A-carriers (59\%).

\section{Analysis of Petting}

Predictors of child-dog petting during the child-pet interaction task were determined via regression analyses using backward selection to obtain a reduced model with the strongest predictors. An initial model included all predictors (demographic covariates, child self-reported relationship qualities, and child genotype). The initial model suggested that petting was associated with child age $\left[F_{(1,84)}=4.18, p=0.044\right]$, OXTR rs53576 genotype $\left[F_{(1,84)}=6.15, p=0.015\right]$, and Negative Interactions with the dog $\left[F_{(1,84)}=6.31, p=0.014\right]$. Specifically, more petting was observed with older child age, OXTR rs53576 A-carrier status, and lower child-reported Negative Interactions with the pet dog (see Figure 1). Petting was not associated with child sex $\left[F_{(1,84)}=0.37\right.$,

TABLE 1 | Principal component analysis (PCA) loadings of dog NRI subscales.

\begin{tabular}{lrr}
\hline & PC1 & PC2 \\
\hline Companionship & $-\mathbf{0 . 4 5}$ & 0.19 \\
Antagonism & 0.14 & $\mathbf{0 . 9 8}$ \\
Intimate & $\mathbf{- 0 . 4 1}$ & -0.05 \\
Nurturance & $\mathbf{- 0 . 4 5}$ & 0.08 \\
Affection & $\mathbf{- 0 . 4 3}$ & 0.01 \\
Reassurance & $\mathbf{- 0 . 4 7}$ & 0.08
\end{tabular}

Loading absolute values $>0.4$ are in bold.

TABLE 2 | Descriptive statistics for child self report relationship qualities and behavioral observation.

\begin{tabular}{lll}
\hline & Mean & SD \\
\hline Children's reported relationships & 3.78 & 0.66 \\
Support from mom & 2.02 & 0.81 \\
Negative interactions with mom & 3.91 & 0.68 \\
Support from dog & 1.52 & 0.81 \\
Negative interactions with dog & 50.00 & \\
Behavioral observation of child pet interaction & 31.04 \\
Percent time spent petting & 19.85 & 14.84 \\
Percent time spent gazing & &
\end{tabular}



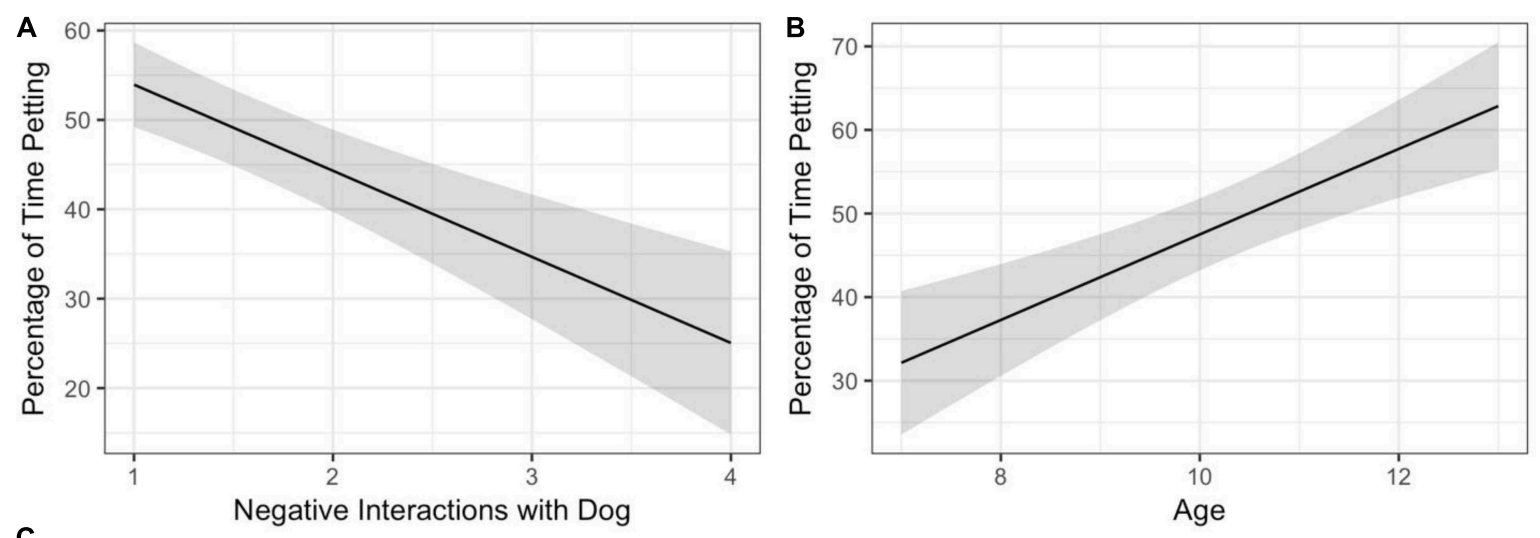

C

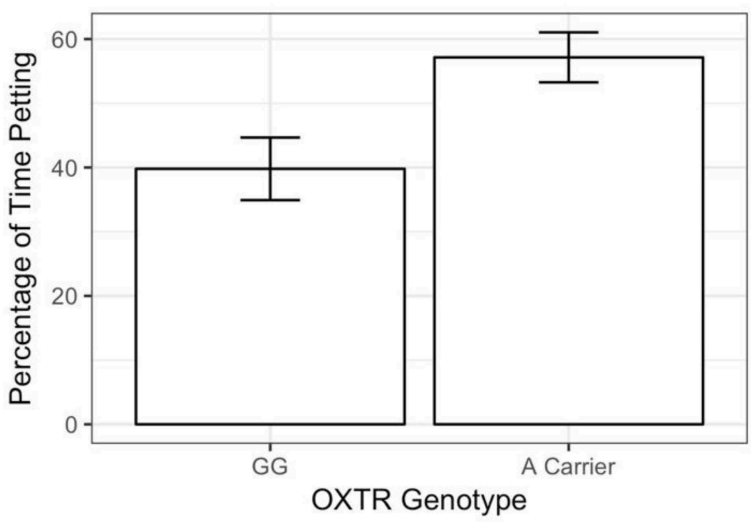

FIGURE 1 | The proportion of time spent petting during a child-dog interaction task was independently predicted by child age, child genotype, and children's self-reported relationship with the pet dog. (A) Higher levels of child-reported negative relationships with the pet dog was associated with less petting. The relationship effect is shown averaged across child genotype and illustrates the overall main effect for the child-pet relationship. The regression line shows the final model prediction with the shaded area indicated standard error of the mean. (B) Older child age is associated with more time spent petting. Lines shows reduced model prediction for each genotype and shading indicates standard error of the mean. (C) Children who are A-carriers at the OXTR rs53576 genetic polymorphism, compared to GG homozygotes, engaged in more petting during the child-pet interaction.

TABLE 3 | Akaike's information criterion (AIC) for each step in the regression predicting petting using backward regression.

\begin{tabular}{|c|c|c|c|c|c|}
\hline Step & $d f$ & Deviance & Residual $d f$ & Residual deviance & AIC \\
\hline Full model & NA & NA & 84 & 69857.28 & 664.21 \\
\hline Dog support & 1 & 9.91 & 85 & 69867.19 & 662.22 \\
\hline Child sex & 1 & 242.34 & 86 & 70109.53 & 660.56 \\
\hline Breed & 5 & 6680.03 & 91 & 76789.56 & 659.39 \\
\hline Negative interaction mom & 1 & 373.48 & 92 & 77163.05 & 657.86 \\
\hline Mom support & 1 & 517.13 & 93 & 77680.18 & 656.51 \\
\hline
\end{tabular}

Values are shown for each variable successively dropped from the model to achieve the best fitting results.

$p=0.54]$, dog breed group $\left[F_{(5,84)}=1.47, p=0.21\right]$, childreported Support from $\operatorname{dog}\left[F_{(1,84)}=1.09, p=0.300\right]$, or either of the two child-reported measures of relationship quality with the mother $\left[F_{(1,84)}=0.53-1.21, p=0.27-0.47\right]$. To obtain the most parsimonious model for our dataset, the model was subjected to backward selection using the Step routine, which identifies the most parsimonious model based on Akaike's information criterion (AIC; see Table 3 for AIC values at each regression step). All three significant predictors - child age, child genotype, and the Negative Interactions dimension of the child-reported relationship measure - were retained in the final model, as shown in Table 4.

\section{Analysis of Gazing}

A comparable regression model was created for gazing during the child-pet interaction. The initial model including all predictors revealed that none of the demographic, genotype, or relationship quality variables was significantly associated with gazing ( $F$ 's $=0.01-2.14, p$ 's $>0.15)$. The backward selection procedure was implemented to yield the most parsimonious 
TABLE 4 | Final regression model of significant predictors of child-dog petting during behavioral observation.

\begin{tabular}{lcccc}
\hline & Estimate & Std. Error & $\boldsymbol{t}$-value & $\boldsymbol{P}$-value \\
\hline Intercept & 4.63 & 23.06 & 0.20 & 0.84 \\
Age & 5.12 & 2.30 & 2.23 & 0.03 \\
OXTR (GG vs. A-carrier) & 12.66 & 6.16 & 2.06 & 0.04 \\
Negative interactions with dog & -9.63 & 3.81 & -2.52 & 0.01 \\
\hline
\end{tabular}

model. The final model of gazing during the child-pet interaction task included only child age as a predictor, however, the association was not statistically significant $(F=2.28, p=0.13$; see Table 5).

\section{DISCUSSION}

The present study was the first to test whether the OXTR genotype and children's perceived relationships with their pet dogs are related to HAI, specifically, petting and gazing. The research design simulated a common, naturally occurring HAI, in which human owners call over their pets, within the context of a controlled laboratory experiment with minimal distractions. On average across children, the total time spent petting was approximately $50 \%$ of the $10 \mathrm{~min}$ interaction period. The results showed that variation at the OXTR polymorphism rs53576 was associated with the proportion of time spent petting during child-pet interactions. Specifically, A-carriers engaged in more petting than children with the GG genotype. This observation is noteworthy given that OXTR rs53576 has previously been suggested as a genetic locus associated with sensitivity to the social environment. Prior research with typically developing children has demonstrated that A-carrier youth are less responsive to parental support (Smearman et al., 2016) and to social consequences of peer relational aggression (Kushner et al., 2017), and show lower levels of interpersonal empathy (Rodrigues et al., 2009; Smith et al., 2014), trust (Krueger et al., 2012), and self-esteem (Saphire-Bernstein et al., 2011). This may be relevant to growing trend of incorporating HAI into behavioral therapy with children for whom human social interactions are challenging (Nimer and Lundahl, 2007; Silva et al., 2018). Although little empirical research has been conducted in this area, there is preliminary evidence that dogs may be preferred social partners for such children. Children with autism, a neurodevelopmental disorder in which social deficits are common, prefer to interact with a dog over another person or toy (Prothmann et al., 2009). Children with anxiety disorders tend to spend long durations interacting with a pet dog but tend to engage in fewer interactions with another person compared to children with other behavioral health problems (Prothmann et al., 2005). Although highly speculative, our results contribute to emerging evidence that pet dogs may be an important source of social interaction for children that have difficulty in other social environments.

The results of this study also demonstrated that children's selfreported negative interactions in the context of their relationship with their pet was related to the proportion of time spent petting the dog. Specifically, higher levels of antagonism, reflecting children's reports that they and their dog hassle each other, annoy each other, and "get on each others' nerves," spent less time engaged with petting. Children's perceptions of support, reflecting items tapping aspects of affection, companionship, and other positive features, were not associated with the proportion of time spent petting. Of note, this was the first study that simultaneously assessed both positive and negative components of children's relationships with their pet dog. Psychometric data from the principal components analysis demonstrated that children's responses about positive and negative relationship qualities were distinct measurable aspects of the child-dog relationship that paralleled the relationship dimensions measured for the child-parent relationship. The observation that negative interactions, and not support, was associated with petting speaks to the need to incorporate both positive and negative aspects of relationships in HAI research.

We also observed that older child age was associated with more time spent petting. This observation was of interest in light of the broad consensus in the developmental literature that, beginning in middle childhood, children spend proportionally less of their social time with parents and more time with other social partners (Lam et al., 2012). Research with 7-10 year old children has shown that with age, children broaden their network of social support figures, including pets (Bryant, 1985). With age, intimate disclosure also declines to parents whereas it rises with other social partners such as peers (Buhrmester and Furman, 1987). Although we did not assess peer relationships in this study, the age effected observed is consistent with the notion that

TABLE 5 | AIC for each step in the regression predicting gazing using backward regression.

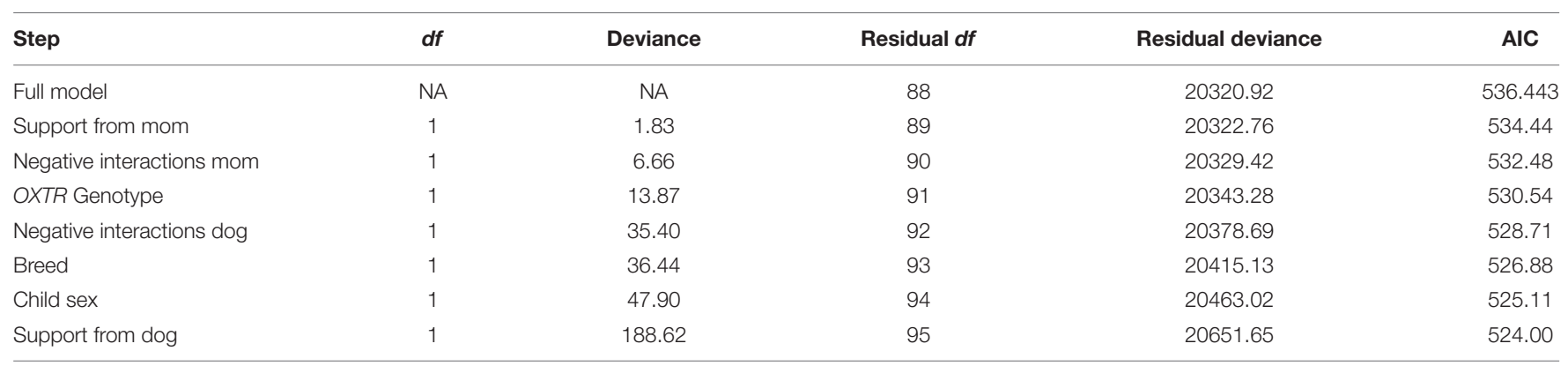

Values are shown for each variable successively dropped from the model. 
non-parental sources of social interaction and support gain in importance during the course of middle childhood, and highlight the role that pets may play in this important developmental transition.

There was no evidence in this study that either genotype or relationship quality was associated with gazing. There are at least two possible explanations for this finding. First, the literature on owner-dog gazing has to date been restricted to research with adults, and there may be unknown differences in childrens' interactions with their pet dogs compared to adult owners. In the absence of any studies comparing adult to child owner's interactions with pets, this possibility cannot be ruled out. Second, in contrast to some studies with adults (e.g., Nagasawa et al., 2015), we did not attempt to manipulate owner-dog gazing, but rather quantified the degree to which such behavior naturally occurred in the context of the child soliciting interaction with the dog. It may be that the amount of naturally occurring gazing (approximately 20\% of the total interaction time) was too low in our behavioral paradigm to detect association with children's individual differences.

The present results should be considered in light of several considerations. First, participants were primarily from nonHispanic White families and thus the generalizability to a more diverse population warrants further study. Second, the participants in this study were typically developing children prescreened for known health conditions. Whether these findings generalize to clinical populations is unknown; however, the present results may serve as foundational research for application to clinical populations. Third, we did not genotype the pet dogs for variation at the OXTR gene. There is some evidence to suggest that dogs' human-directed behavior is associated with genetic polymorphisms at OXTR (Kubinyi et al., 2017; Oláh et al., 2017) or OXTR methylation (Cimarelli et al., 2017). Genotyping OXTR in both children and the pet dogs may reveal more nuanced associations of OXTR genotype within the context of HAIs. Fourth, this study focused on families who already owned a pet dog. This was intentional to avoid the inherent challenge of interpreting child-dog interaction among a mixed group of dog owners vs. non-owners. Finally, the study was conducted in a research laboratory. It is possible that childdog interaction in a laboratory environment may not be the same as in more naturalistic environments. This limitation is

\section{REFERENCES}

Anderson, K. L., and Olson, M. R. (2006). The value of a dog in a classroom of children with severe emotional disorders. Anthrozoos A Multidiscip. J. Interact. People Anim. 19, 35-49. doi: 10.2752/089279306785593919

Barker, S. B., Knisely, J. S., McCain, N. L., and Best, A. M. (2005). Measuring stress and immune response in healthcare professionals following interaction with a therapy dog: a pilot study. Psychol. Rep. 96, 713-729. doi: 10.2466/pr0.96.3. 713-729

Barrera, G., Jakovcevic, A., Elgier, A. M., Mustaca, A., and Bentosela, M. (2010). Responses of shelter and pet dogs to an unknown human. J. Vet. Behav. Clin. Appl. Res. 5, 339-344. doi: 10.1016/j.jveb.2010.08.012

Belsky, J., Jonassaint, C., Pluess, M., Stanton, M., Brummett, B., and Williams, R. (2009). Vulnerability genes or plasticity genes? Mol. Psychiatry 14, 746-754. doi: $10.1038 / \mathrm{mp} .2009 .44$ offset, however, by the benefits of the tightly controlled context of a laboratory, with standardized environmental and testing conditions for maximizing the validity of the observed results and reducing distractions and confounding variables. Moreover, the direct behavioral observation of child-pet interaction lends higher confidence in the validity of the observed empirical associations compared to descriptive or self-report studies.

This study adds to the growing body of literature on HAI by documenting two key factors that predict natural variation in children's interactions with their pet dogs. This knowledge is critical as the field as a whole strives to maximize the potential therapeutic benefits of HAI for clinical populations. A greater understanding of the individual differences that influence children's interactions with familiar animals will also aid in the broader research goal of determining the potential benefits and challenges of pet ownership for children.

\section{AUTHOR CONTRIBUTIONS}

DK developed the study, oversaw its execution, analyzed the data, and wrote the manuscript. NH collected the data, conducted the behavioral coding, and analyzed the data. SB conducted the genotyping and analyzed the data. All authors contributed to the writing of the manuscript and approved the final manuscript.

\section{FUNDING}

This research was supported by the National Institutes of Health grant HD071288 to DK. The content is solely the responsibility of the authors and does not necessarily represent the official views of the funders.

\section{ACKNOWLEDGMENTS}

We would like to thank Clive Wynne, Jingwen Liu, and Natalie Hadad for their contributions to the research from which this study was developed. We gratefully acknowledge the families who participated in this study, without whom this research would not have been possible.

Bryant, B. K. (1985). The neighborhood walk: sources of support in middle childhood. Monogr. Soc. Res. Child Dev. 50, 1-122. doi: 10.2307/3333847

Buhrmester, D., and Furman, W. (1987). The development of companionship and intimacy. Child Dev. 58, 1101-1113. doi: 10.2307/1130550

Carter, C. S. (2014). Oxytocin pathways and the evolution of human behavior. Annu. Rev. Psychol. 65, 17-39. doi: 10.1146/annurev-psych-010213-115110

Charnetski, J. C., Riggers, S., and Brennan, F. X. (2004). Effect of petting a dog on immune system function. Psychol. Rep. 95, 1087-1091. doi: 10.2466/pr0.95.3f. 1087-1091

Cimarelli, G., Virányi, Z., Turcsán, B., Rónai, Z., Sasvári-Székely, M., and Bánlaki, Z. (2017). Social behavior of pet dogs is associated with peripheral OXTR methylation. Front. Psychol. 8:549. doi: 10.3389/fpsyg.2017.00549

Cromer, L. D., and Barlow, M. R. (2013). Factors and convergent validity of the pet attachment and life impact scale (PALS). Hum. Anim. Interact. Bull. 1, 34-56. doi: $10.1037 /$ e634302013-004 
Daly, B., and Morton, L. L. (2006). An investigation of human-animal interactions and empathy as related to pet preference, ownership, attachment, and attitudes in children. Anthrozoos A Multidiscip. J. Interact. People Anim. 19, 113-127. doi: $10.2752 / 089279306785593801$

Demello, L. R. (1999). The effect of the presence of a companion-animal on physiological changes following the termination of cognitive stressors. Psychol. Health 14, 859-868. doi: 10.1080/08870449908407352

DeRosier, M., and Kupersmidt, J. (1991). Costa rican children's perceptions of their social networks. Dev. Psychol. 27, 656-662. doi: 10.1037/0012-1649.27.4.656

Friedmann, E., Katcher, A., Thomas, S. A., Lynch, J. J., and Messent, P. R. (1983). Social interaction and blood pressure: influence of animal companions. J. Nerv. Ment. Dis. 171, 461-465. doi: 10.1097/00005053-198308000-00002

Furman, W., and Buhrmester, D. (1992). Age and sex differences in perceptions of networks of personal relationships. Child Dev. 63, 103-115. doi: 10.2307/ 1130905

Furman, W., and Burmheister, D. (1985). Children's perceptions of the personal relationships in their social networks. Dev. Psychol. 21, 1016-1024. doi: 10.1037/ 0012-1649.21.6.1016

Griffin, J., McCune, S., Malholmes, V., and Hurley, K. (2011). "Scientific research on human-animal interaction," in Animals in Our Lives: Human-Animal Interaction in Family, Community, and Therapeutic Settings, eds P. McCardle, S. McCune, J. A. Griffin, L. Esposito, and L. Freund (Baltimore MD: Paul H. Brooks Publishing Co.), 227-236.

Hall, N., Liu, J., Kertes, D. A., and Wynne, C. D. L. (2016). Behavioral and self-report measures influencing children's reported attachment to their dog. Anthrozoös 29, 137-150. doi: 10.1080/08927936.2015.1088683

Handlin, L., Hydbring-Sandberg, E., Nilsson, A., Ejdebäck, M., Jansson, A., and Uvnäs-Moberg, K. (2011). Short-term interaction between dogs and their owners: effects on oxytocin, cortisol, insulin and heart rate-an exploratory study. Anthrozoos 24, 301-315. doi: 10.2752/175303711X13045914865385

Insel, T. R. (1992). Oxytocin - A neuropeptide for affiliation: evidence from behavioral, receptor autoradiographic, and comparative studies. Psychoneuroendocrinology 17, 3-35. doi: 10.1016/0306-4530(92)90073-G

Jakovcevic, A., Mustaca, A., and Bentosela, M. (2012). Do more sociable dogs gaze longer to the human face than less sociable ones? Behav. Process. 90, 217-222. doi: 10.1016/j.beproc.2012.01.010

Jenkins, J. L. (1986). Physiological effects of petting a companion animal. Psychol. Rep. 58, 21-22. doi: 10.2466/pr0.1986.58.1.21

Johnson, T. P., Garrity, T. F., and Stallones, L. (1992). Psychometric evaluation of the lexington attachment to pets scale (LAPS). Anthrozoös 5, 160-175. doi: 10.2752/089279392787011395

Kerns, K. A., Stuart-Parrigon, K. L., Coifman, K. G., van Dulmen, M. H. M., and Koehn, A. (2018). Pet dogs: does their presence influence preadolescents' emotional responses to a social stressor? Soc. Dev. 27, 34-44. doi: 10.1111/sode. 12246

Kertes, D. A., Liu, J., Hall, N., Hadad, N., Wynne, C., and Bhatt, S. S. (2017). Effect of pet dogs on children's perceived stress and cortisol stress response. Soc. Dev. 26, 382-401. doi: 10.1111/sode.12203

Kim, H. S., Sherman, D. K., Sasaki, J. Y., Xu, J., Chu, T. Q., Ryu, C., et al. (2010). Culture, distress, and oxytocin receptor polymorphism (OXTR) interact to influence emotional support seeking. Proc. Natl. Acad. Sci. 107, 15717-15721. doi: 10.1073/pnas.1010830107

Kirsch, P., Esslinger, C., Chen, Q., Mier, D., Lis, S., Siddhanti, S., et al. (2005). Oxytocin modulates neural circuitry for social cognition and fear in humans. J. Neurosci. 25, 11489-11493. doi: 10.1523/JNEUROSCI.3984-05.2005

Kotrschal, K., and Ortbauer, B. (2003). Behavioral effects of the presence of a dog in a classroom. Anthrozoös 16, 147-159. doi: 10.2752/089279303786992170

Krueger, F., Parasuraman, R., Iyengar, V., Thornburg, M., Weel, J., Lin, M., et al. (2012). Oxytocin receptor genetic variation promotes human trust behavior. Front. Hum. Neurosci. 6:4. doi: 10.3389/fnhum.2012.00004

Kubinyi, E., Bence, M., Koller, D., Wan, M., Pergel, E., Ronai, Z., et al. (2017). Oxytocin and opioid receptor gene polymorphisms associated with greeting behavior in dogs. Front. Psychol. 8:1520. doi: 10.3389/fpsyg.2017.01520

Kurdek, L. A. (2008). Pet dogs as attachment figures. J. Soc. Pers. Relat. 25, 247-266. doi: $10.1177 / 0265407507087958$

Kushner, S. C., Herzhoff, K., Vrshek-Schallhorn, S., and Tackett, J. L. (2017). Depression in early adolescence: contributions from relational aggression and variation in the oxytocin receptor gene. Aggress. Behav. 44, 60-68. doi: 10.1002/ ab. 21724

Lam, C. B., McHale, S. M., and Crouter, A. C. (2012). Parent-child shared time from middle childhood to late adolescence: developmental course and adjustment correlates. Child Dev. 83, 2089-2103. doi: 10.1111/j.1467-8624. 2012.01826.x

Lim, M. M., and Young, L. J. (2006). Neuropeptidergic regulation of affiliative behavior and social bonding in animals. Horm. Behav. 50, 506-517. doi: 10. 1016/j.yhbeh.2006.06.028

Lucht, M. J., Barnow, S., Sonnenfeld, C., Rosenberger, A., Grabe, H. J., Schroeder, W., et al. (2009). Associations between the oxytocin receptor gene $(O X T R)$ and affect, loneliness and intelligence in normal subjects. Prog. Neuropsychopharmacol. Biol. Psychiatry 33, 860-866. doi: 10.1016/j.pnpbp. 2009.04.004

Luo, S., and Han, S. (2014). The association between an oxytocin receptor gene polymorphism and cultural orientations. Cult. Brain 2, 89-107. doi: 10.1007/ s40167-014-0017-5

Moilanen, K. L., and Raffaelli, M. (2010). Support and conflict in ethnically diverse young adults' relationships with parents and friends. Int. J. Behav. Dev. 34, 46-52. doi: 10.1177/0165025409348553

Nagasawa, M., Kikusui, T., Onaka, T., and Ohta, M. (2009). Dog's gaze at its owner increases owner's urinary oxytocin during social interaction. Horm. Behav. 55, 434-441. doi: 10.1016/j.yhbeh.2008.12.002

Nagasawa, M., Mitsui, S., En, S., Ohtani, N., Ohta, M., Sakuma, Y., et al. (2015). Oxytocin-gaze positive loop and the coevolution of human-dog bonds. Science 348, 333-336. doi: 10.1126/science. 1261022

Neumann, I. D., Wigger, A., Torner, L., Holsboer, F., and Landgraf, R. (2000). Brain oxytocin inhibits basal and stress-induced activity of the hypothalamopituitary-adrenal axis in male and female rats: partial action within the paraventricular nucleus. J. Neuroendocrinol. 12, 235-243. doi: 10.1046/j.13652826.2000.00442.x

Nimer, J., and Lundahl, B. (2007). Animal-assisted therapy: a meta-analysis. Anthrozoos A Multidiscip. J. Interact. People Anim. 20, 225-238. doi: 10.2752/ $089279307 \mathrm{X} 224773$

Odendaal, J. S. J., and Meintjes, R. A. (2003). Neurophysiological correlates of affiliative behaviour between humans and dogs. Vet. J. 165, 296-301. doi: 10. 1016/S1090-0233(02)00237-X

Odendaal, J. S. J. (2000). Animal-assisted therapy - magic or medicine? J. Psychosom. Res. 49, 275-280. doi: 10.1016/S0022-3999(00)00183-5

Oláh, K., Topál, J., Kovács, K., Kis, A., Koller, D., Park, S. Y., et al. (2017). Gaze-following and reaction to an aversive social interaction have corresponding associations with variation in the OXTR gene in dogs but not in human infants. Front. Psychol. 8:2156. doi: 10.3389/fpsyg.2017. 02156

Prothmann, A., Albrecht, K., Dietrich, S., Hornfeck, U., Stieber, S., and Ettrich, C. (2005). Analysis of child-dog play behavior in child psychiatry. Anthrozoös 18, 43-58. doi: 10.2752/089279305785594261

Prothmann, A., Ettrich, C., and Prothmann, S. (2009). Preference for, and responsiveness to, people, dogs and objects in children with autism. Anthrozoös 22, 161-171. doi: 10.2752/175303709X434185

R Core Team (2016) R: A Language and Environment for Statistical Computing. Austria: R Foundation for Statistical Computing. Availabel at: https://www.Rproject.org/

Rodrigues, S. M., Saslow, L. R., Garcia, N., John, O. P., and Keltner, D. (2009). Oxytocin receptor genetic variation relates to empathy and stress reactivity in humans. Proc. Natl. Acad. Sci. U.S.A. 106, 21437-21441. doi: 10.1073/pnas. 0909579106

Saphire-Bernstein, S., Way, B. M., Kim, H. S., Sherman, D. K., and Taylor, S. E. (2011). Oxytocin receptor gene (OXTR) is related to psychological resources. Proc. Natl. Acad. Sci. U.S.A. 108, 15118-15122. doi: 10.1073/pnas.1113137108

Serpell, J. A. (1996). Evidence for an association between pet behavior and owner attachment levels. Appl. Anim. Behav. Sci. 47, 49-60. doi: 10.1016/01681591(95)01010-6

Shantz, C. U., and Hartup, W. W. (1992). "Conflict in child and adolescent development," in Cambridge Studies in Social and Emotional Development, eds C. U. Shantz and W. W. Hartup (New York, NY: Cambridge University Press). 
Shiloh, S., Sorek, G., and Terkel, J. (2003). Reduction of state-anxiety by petting animals in a controlled laboratory experiment. Anxiety Stress Coping 16, 387-395. doi: 10.1080/1061580031000091582

Silva, K., Lima, M., Santos-Magalhães, A., Fafiães, C., and de Sousa, L. (2018). Can dogs assist children with severe autism spectrum disorder in complying with challenging demands? an exploratory experiment with a live and a robotic dog. J. Altern. Complement. Med. 24, 238-242. doi: 10.1089/acm.2017. 0254

Smearman, E. L., Yu, T., and Brody, G. H. (2016). Variation in the oxytocin receptor gene moderates the protective effects of a family-based prevention program on telomere length. Brain Behav. 6, 1-12. doi: 10.1002/brb3.423

Smith, K. E., Porges, E. C., Norman, G. J., Connelly, J. J., and Decety, J. (2014). Oxytocin receptor gene variation predicts empathic concern and autonomic arousal while perceiving harm to others. Soc. Neurosci. 9, 1-9. doi: 10.1080/ 17470919.2013.863223

Vagnoli, L., Caprilli, S., Vernucci, C., Zagni, S., Mugnai, F., and Messeri, A. (2015). Can presence of a dog reduce pain and distress in children during venipuncture? Pain Manag. Nurs. 16, 89-95. doi: 10.1016/j.pmn.2014. 04.004
Van Horn, K. R., and Cunegatto, M. J. (2000). Interpersonal relationships in Brazilian adolescents. Int. J. Behav. Dev. 24, 199-203. doi: 10.1080/ 016502500383322

Vormbrock, J. K., and Grossberg, J. M. (1988). Cardiovascular effects of humanpet dog interactions. J. Behav. Med. 11, 509-517. doi: 10.1007/BF0084 4843

Wilson, C. C. and Barker, S. B. (2003). Challenges in designing human-animal interaction research. Am. Behav. Sci. 47, 16-28. doi: 10.1177/0002764203255208

Conflict of Interest Statement: The authors declare that the research was conducted in the absence of any commercial or financial relationships that could be construed as a potential conflict of interest.

Copyright (c) 2018 Kertes, Hall and Bhatt. This is an open-access article distributed under the terms of the Creative Commons Attribution License (CC BY). The use, distribution or reproduction in other forums is permitted, provided the original author(s) and the copyright owner(s) are credited and that the original publication in this journal is cited, in accordance with accepted academic practice. No use, distribution or reproduction is permitted which does not comply with these terms. 